\title{
Numerical and Experimental Research of Heat and Mass Transfer at the Heterogeneous System Ignition by Local Energy Source with Limited Heat Content
}

\author{
Dmitrii O. Glushkov, Genii V. Kuznetsov, and Pavel A. Strizhak \\ National Research Tomsk Polytechnic University, 30 Lenin Avenue, Tomsk 634050, Russia \\ Correspondence should be addressed to Dmitrii O. Glushkov; dmitriyog@tpu.ru
}

Received 14 March 2014; Accepted 5 June 2014; Published 24 June 2014

Academic Editor: Zhijun Zhang

Copyright (C) 2014 Dmitrii O. Glushkov et al. This is an open access article distributed under the Creative Commons Attribution License, which permits unrestricted use, distribution, and reproduction in any medium, provided the original work is properly cited.

\begin{abstract}
Numerical and experimental investigations were executed for determination of macroscopic regularities of heat and mass transfer processes under the conditions of the phase transformations and chemical reaction at the ignition of vapors coming from fabrics impregnated by combustible liquid into oxidant area at the local power supply. It was established that initial temperature $\Theta_{p}>1$ of local energy source and volume fraction $\varphi>30 \%$ of combustible liquid vapors in fabric are necessary for realization of ignition conditions in a system "fabric_combustible liquid_oxidant.". Thus three ignition modes are possible for such system. The most widespread mode is an arrangement of ignition zone near the lateral side of local energy source. Also we obtained approximating expressions of ignition delay time on initial temperature and characteristic size of a local energy source for fabrics impregnated by some kinds of combustible liquids (gasoline, kerosene, and diesel fuel). Its dependences may be useful at engineering calculations of fire danger for processes of single hot particles interaction with liquid combustible substances.
\end{abstract}

\section{Introduction}

Numerical and experimental investigations of ignition processes for solid [1-4] and liquid [5-8] condensed substances, polymer materials [9-11], and gas-vapor mixtures [12-14] by the energy source with limited power consumption (metallic and nonmetallic particles, wires, cores, concentrate radiation flows, etc.) were held in recent years. Investigation results allowed defining extreme and optimum ignition conditions of high-energy materials under the local heating for the most important practical supplements (special equipment, fire and explosion safety of substances and materials, chemical industry, and others).

In power engineering and mechanical engineering at various technological processes products from fabric rather and often can be applied to cleaning surfaces, putting greasing, removing surplus of greasing, and so forth. As a rule, such fabrics after corresponding technological processes have high fire danger. The numerical investigation results of regularities for heterogeneous systems (fabrics impregnated by combustible liquid) ignition by the local energy source are reduced in [15]. The characteristic fabric thicknesses were chosen essentially bigger than heating source rates. Because of the established [15] integrated ignition characteristics (especially ignition delay time $t_{d}$ ) can be considered as high values.

Actually realization of other conditions is possible. More often local heating sources (particles, wires, cores, shavings and etc.) have rates equitable or even exceeding the thickness of fabrics impregnated by combustion liquid. Numerical and experimental investigations of ignition conditions for such structures are interesting.

The aim of the present work is to research numerically and experimentally the macroscopic regularities and extreme ignition conditions of typical liquid combustible substances (fuels) vapors coming from fabric surfaces impregnated by it (fabric thicknesses equitable with hot particle rates) under the local heating. 


\section{Problem Statement}

The problem statement is similar as in the work [15]. The system "hot particle-fabric impregnated by combustible liquid-gas mixture" was considered (Figure 1). Unlike the problem [15] fabric thickness $Z_{1}$ in present work is comparable with characteristic rates of energy source (hot particle) $R_{p}$ $\left(R_{p}=R_{1}\right)$ and $Z_{p}\left(Z_{p}=Z_{2}-Z_{1}\right)$. We considered $2 \mathrm{D}$ problem statement for heat and mass transfer processes investigation in decision area (Figure 1). Though, it was established that the difference between similar integrated characteristics at 2D and $3 \mathrm{D}$ problem statements makes less than $10 \%$ according to analysis of numerical research results [16]. Nevertheless at 3D statement the mathematical model is more difficult and calculations are more complex than at $2 \mathrm{D}$ statement.

It was shown that at initial moment $(\tau=0)$ fabric is impregnated by liquid. Its volume fraction $(\varphi)$ was known. Components of combustible liquid start evaporating intensively when near-surface layer was heated up to high temperatures by energy of particle fallen on the fabric surface. Generated fuel vapors mix with oxidant (air) is heated by the particle energy. At the critical temperature and concentration of gas mixture ignition happened.

The investigations were held for liquid fuels (gasoline, kerosene, and diesel fuel) and widespread fabrics (wool, silk, and flax). The steel particle in the shape of disk with small rates $R_{p}$ and $Z_{p}$ was chosen as a source of heat. The characteristic rates of domains $R_{L}$ and $Z_{L}$ for ignition problem solution were taken far more than $R_{p}$ and $Z_{p}$ (Figure 1).

Numerical simulation was held with the following assumptions.

(1) The substance with known characteristics appeared as a result of evaporation. The "effective" values of activation energy $E$ and preexponential factor $k_{0}$ are usually [17] determined in experimental research of kinetic parameters for oxidation reaction of liquid vapors. The realization of one "effective" reaction is supposed, in which one substance participates. Appropriately, the theoretical analyses of investigated process are possible when kinetic scheme, for which values $E$ and $k_{0}$ are known, is used.

(2) The thermophysical characteristics of particle, fabric, liquid, and air substances are constant. It is established that for investigating range of temperatures in system "hot particle-fabric impregnated by combustible liquid-gas mixture" (Figure 1) thermophysical characteristics changing depend on interacting substances temperature insignificantly.

(3) The contact between the particle and the fabric is perfect. Numerical and experimental investigations $[3,4]$ showed that the particle and condensed substance surfaces do not deform at the small rates (less than $1.5 \mathrm{~m} / \mathrm{s}$ ) of particle movement.

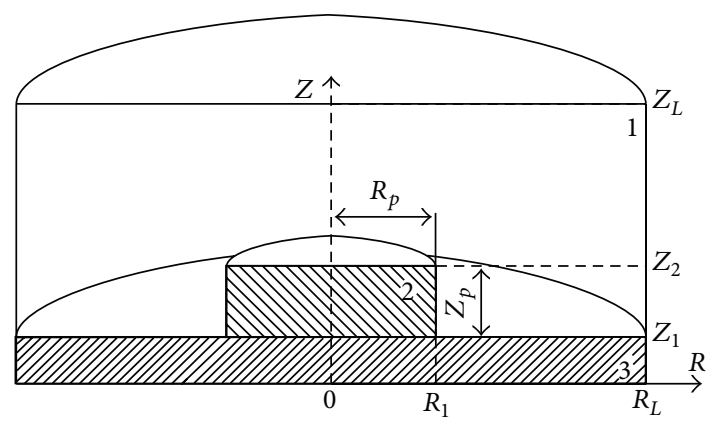

FIgURE 1: A scheme of the solution domain of the problem at $0<\tau<$ $\tau_{d}: 1$-gas mixture, 2-metal particle, and 3-fabric impregnated by liquid combustible substance.

The ignition conditions for the heat and mass transfer model were the following [18].

(1) The heat released as a result of chemical reaction of gas oxidation in air exceeds that transferred from the particle to the substance and the gas mixture.

(2) The gas mixture temperature is higher than the initial temperature of the hot steel particle.

At the numerical research the main integrated characteristic ignition delay time was defined as time period from the moment of contact between hot particle and fabric surface to the moment of formulated ignition conditions performance.

\section{Mathematical Model}

The mathematical model describes processes of thermal conduction in hot steel particle and fabric at their interconnection, crystallization of particle material at its cooling, evaporation of combustible liquid at heating near-surface layer of fabric impregnated by liquid fuel, thermal convection and diffusion of evaporation products (combustible gases) at their mixing with air, and oxidation of gas mixture at its heating. The set of nonlinear nonstationary differential equations $[17,19-22]$ at $0<\tau<\tau_{d}$ is follows:

$$
\begin{array}{ll}
Z_{1}<Z<Z_{2}, & R_{1}<R<R_{L} ; \\
Z_{2}<Z<Z_{L}, & 0<R<R_{L}
\end{array}
$$

the equation of gas mixture (evaporation products and air) movement is

$$
\begin{aligned}
& \frac{1}{\text { Sh }} \frac{\partial \Omega}{\partial \tau}+U \frac{\partial \Omega}{\partial R}+V \frac{\partial \Omega}{\partial Z}-U \frac{\Omega}{R} \\
& \quad=\frac{1}{\operatorname{Re}}\left[\frac{\partial^{2} \Omega}{\partial R^{2}}+\frac{1}{R} \frac{\partial \Omega}{\partial R}+\frac{\partial^{2} \Omega}{\partial Z^{2}}-\frac{\Omega}{R^{2}}\right]+\frac{\partial \Theta_{1}}{\partial R} ;
\end{aligned}
$$

Poisson's equation is

$$
\frac{\partial^{2} \Psi}{\partial R^{2}}-\frac{1}{R} \frac{\partial \Psi}{\partial R}+\frac{\partial^{2} \Psi}{\partial Z^{2}}=-R \Omega
$$


the thermal conduction equation for gas mixture considering oxidation reaction is

$$
\begin{aligned}
\frac{1}{\operatorname{Sh}} & \frac{\partial \Theta_{1}}{\partial \tau}+U \frac{\partial \Theta_{1}}{\partial R}+V \frac{\partial \Theta_{1}}{\partial Z} \\
& =\frac{1}{\operatorname{RePr}}\left[\frac{\partial^{2} \Theta_{1}}{\partial R^{2}}+\frac{1}{R} \frac{\partial \Theta_{1}}{\partial R}+\frac{\partial^{2} \Theta_{1}}{\partial Z^{2}}\right]+\frac{Q_{o} W_{o} z_{L}}{\rho_{1} C_{1} \Delta T V_{m}} ;
\end{aligned}
$$

the thermal diffusion equation for evaporation products is

$$
\begin{aligned}
& \frac{1}{\operatorname{Sh}} \frac{\partial C_{f}}{\partial \tau}+U \frac{\partial C_{f}}{\partial R}+V \frac{\partial C_{f}}{\partial Z} \\
& \quad=\frac{1}{\operatorname{ReSc}}\left[\frac{\partial^{2} C_{f}}{\partial R^{2}}+\frac{1}{R} \frac{\partial C_{f}}{\partial R}+\frac{\partial^{2} C_{f}}{\partial Z^{2}}\right]-\frac{z_{L} W_{o}}{\rho_{4} V_{m}} ;
\end{aligned}
$$

the balance equation for mass conservation law is

$$
\begin{gathered}
C_{f}+C_{o}=1 ; \\
Z_{1}<Z<Z_{2}, \quad 0<R<R_{1}
\end{gathered}
$$

the thermal conduction equation for metallic particle considering its material crystallization is

$$
\begin{array}{r}
\frac{1}{\mathrm{Fo}_{2}} \frac{\partial \Theta_{2}}{\partial \tau}=\frac{\partial^{2} \Theta_{2}}{\partial R^{2}}+\frac{1}{R} \frac{\partial \Theta_{2}}{\partial R}+\frac{\partial^{2} \Theta_{2}}{\partial Z^{2}}+\frac{Q_{c} W_{c} z_{L}}{z_{p} \Delta T \lambda_{2}} \\
0<Z<Z_{1}, \quad 0<R<R_{L} ;
\end{array}
$$

the thermal conduction equation for fabric impregnated by combustible liquid is

$$
\frac{1}{\mathrm{Fo}_{3}} \frac{\partial \Theta_{3}}{\partial \tau}=\frac{\partial^{2} \Theta_{3}}{\partial R^{2}}+\frac{1}{R} \frac{\partial \Theta_{3}}{\partial R}+\frac{\partial^{2} \Theta_{3}}{\partial Z^{2}} .
$$

Dimensionless complexes are

$$
\begin{gathered}
\operatorname{Sh}=\frac{V_{m} t_{m}}{z_{L}}, \quad \operatorname{Re}=\frac{V_{m} z_{L}}{v}, \\
\operatorname{Pr}=\frac{v \rho C}{\lambda}, \quad \mathrm{Sc}=\frac{v}{D}, \quad \mathrm{Fo}=\frac{\lambda t_{m}}{\rho C z_{L}^{2}} .
\end{gathered}
$$

We set temperatures of hot particle, fabric impregnated by combustible liquid and air, fuel vapors concentration in gas mixture, values of stream function, and vortex velocity vector as initial conditions $(\tau=0)$ :

(i) $0<R<R_{L}, 0<Z<Z_{1}$ :

$$
\Theta_{3}=\Theta_{0}
$$

(ii) $0<R<R_{1}, Z_{1}<Z<Z_{2}$ :

$$
\Theta_{2}=\Theta_{p}
$$

(iii) $R_{1}<R<R_{L}, Z_{1}<Z<Z_{2} ; 0<R<R_{L}, Z_{2}<Z<Z_{L}$ :

$$
\Theta_{1}=\Theta_{0}, \quad C_{f}=0, \quad \Psi=0, \quad \Omega=0 .
$$

We used boundary perfect thermal contact conditions, taking into account liquid evaporation and condition of equality to zero gradients of corresponding functions at the statement of boundary conditions $\left(0<\tau \leq \tau_{d}\right)$ :

(i) $R=0, R=R_{L}, 0<Z<Z_{1}$ :

$$
\frac{\partial \Theta_{3}}{\partial R}=0
$$

(ii) $R=0, Z_{1}<Z<Z_{2}$ :

$$
\frac{\partial \Theta_{2}}{\partial R}=0
$$

(iii) $R=0, Z_{2}<Z<Z_{L} ; R=R_{L}, Z_{1}<Z<Z_{L}$ :

$$
\frac{\partial \Theta_{1}}{\partial R}=0, \quad \frac{\partial C_{f}}{\partial R}=0, \quad \frac{\partial \Psi}{\partial R}=0 ;
$$

(iv) $R=R_{1}, Z_{1}<Z<Z_{2}$ :

$$
\begin{aligned}
& \frac{\partial \Theta_{2}}{\partial R}=\left(\frac{\lambda_{1}}{\lambda_{2}}\right)\left(\frac{\partial \Theta_{1}}{\partial R}\right), \quad \Theta_{2}=\Theta_{1}, \\
& \frac{\partial C_{f}}{\partial R}=0, \quad \frac{\partial \Psi}{\partial R}=0, \quad \Psi=0 ;
\end{aligned}
$$

(v) $Z=0,0<R<R_{L}$ :

$$
\frac{\partial \Theta_{3}}{\partial Z}=0
$$

(vi) $Z=Z_{1}, 0<R<R_{1}$ :

$$
\frac{\partial \Theta_{3}}{\partial Z}=\left(\frac{\lambda_{2}}{\lambda_{3}}\right)\left(\frac{\partial \Theta_{2}}{\partial Z}\right), \quad \Theta_{3}=\Theta_{2} ;
$$

(vii) $Z=Z_{1}, R_{1}<R<R_{L}$ :

$$
\begin{gathered}
\frac{\partial \Theta_{3}}{\partial Z}=\left(\frac{\lambda_{1}}{\lambda_{3}}\right)\left(\frac{\partial \Theta_{1}}{\partial Z}\right)-\frac{Q_{e} W_{e} z_{L}}{\Delta T \lambda_{3}}, \\
\Theta_{3}=\Theta_{1},
\end{gathered}
$$

$$
\frac{\partial C_{f}}{\partial Z}=-\frac{W_{e} z_{L}}{\rho_{1} D_{1}}, \quad \frac{\partial \Psi}{\partial Z}=U, \quad-\frac{\partial \Psi}{\partial X}=V ;
$$

(viii) $Z=Z_{2}, 0<R<R_{1}$ :

$$
\begin{aligned}
& \frac{\partial \Theta_{2}}{\partial Z}=\left(\frac{\lambda_{1}}{\lambda_{2}}\right)\left(\frac{\partial \Theta_{1}}{\partial Z}\right), \quad \Theta_{2}=\Theta_{1}, \\
& \frac{\partial C_{f}}{\partial Z}=0, \quad \frac{\partial \Psi}{\partial Z}=0, \quad \Psi=0 ;
\end{aligned}
$$

(ix) $Z=Z_{L}, 0<R<R_{L}$ :

$$
\frac{\partial \Theta_{1}}{\partial Z}=0, \quad \frac{\partial C_{f}}{\partial Z}=0, \quad \frac{\partial \Psi}{\partial Z}=0 .
$$


The transition to the dimensionless variables $\left(R=r / z_{L}\right.$, $Z=z / z_{L}, \tau=t / t_{m}, U=u / V_{m}, V=v / V_{m}, V_{m}=\sqrt{g \beta \Delta T z_{L}}$, $\Theta=\left(T-T_{0}\right) / \Delta T, \Psi=\psi / \psi_{0}, \Omega=\omega / \omega_{0}, \psi_{0}=V_{m} z_{L}^{2}$, and $\left.\omega_{0}=V_{m} / z_{L}\right)$ was performed for the following scale values: characteristic size of solution area $z_{L}=0.02 \mathrm{~m}$; time scale $t_{m}=$ $1 \mathrm{~s}$; temperature scale $T_{m}=1000 \mathrm{~K}$; gravitational acceleration $g=9.8 \mathrm{~m} / \mathrm{s}^{2}$.

The mass rate of gas mixture oxidation [17] is

$$
W_{o}=\rho_{1} k_{0} C_{f} C_{o} \exp \left[-\frac{E}{R_{t} T_{1}}\right] .
$$

The mass rate of combustible liquid evaporation at heating near-surface layer of fabric impregnated by it is

$$
W_{e}=\frac{A\left(P^{n}-P\right)}{\sqrt{2 \pi R_{t} T_{\mathrm{dr}} / M}} .
$$

Accommodation coefficient for process of combustible liquid evaporation is

$$
A=\frac{35}{\left(P^{n}\right)^{0.56}} .
$$

The mass rate of particle material crystallization at its cooling is

$$
W_{c}=V_{c} \rho_{2}
$$

The linear rate of particle material crystallization is

$$
V_{c}=\frac{\delta(x, y, t+\Delta t)+\delta(x, y, t)}{\Delta t},
$$

where $\delta(x, y, t+\Delta t), \delta(x, y, t)$, is distances from the bottom side of a particle to the crystallization front on $(t+\Delta t)$ th and $t$ th time steps, $\mathrm{m}$.

The system of (2)-(8) with initial and boundary conditions was solved by the finite difference method. To solve difference analogs of differential equations the locally one-dimensional method was applied. Nonlinear difference analogs of differential equations were solved by the iteration method. To solve one-dimensional differential equations the double sweep method with the implicit four-point scheme was applied. We selected no less than 400 knots of the difference net for each of the coordinates and used time step $10^{-6} \mathrm{~s}$.

The reliability of the obtained results was verified by the comparison with the experimental data. Besides check of the energy conservation law in the solution field was carried out according to algorithm given in $[5,6,8]$. The error of the energy conservation law at change of initial temperature and the rates of a hot particle did not exceed $2.5 \%$.

\section{Experimental Method}

At the experimental investigations we used the plant (Figure 2) and methods described in [23]. The single metallic disk with different rates of radius $R_{p}=0.15-0.25$ and height $Z_{p}=0.15-0.25$ (Figure 1) was used as a heat source at various

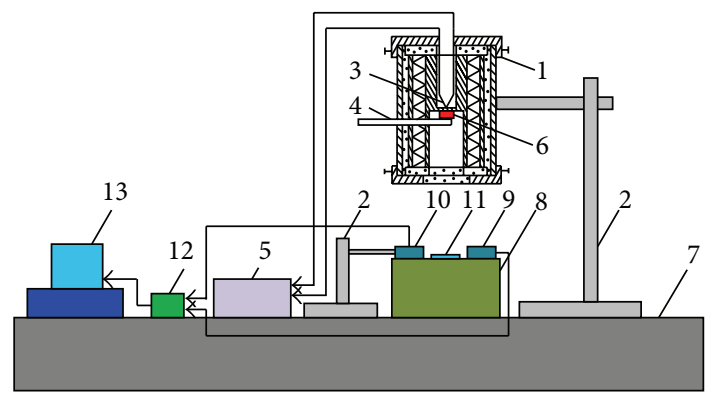

FIGURE 2: Schematic diagram of experimental installation: 1heating device, 2-support, 3-thermocouple, 4-ceramic core, 5temperature sensor, 6-metal particle, 7-basis of experimental installation, 8-fire-resistant platform, 9 and 10-flame registration system (radiation source and detector), 11-fabric impregnated by liquid combustible substance, 12-analog to digital converter, and 13-computer.

series of experiments. The values of disk height $\left(Z_{p}\right)$ and radius $\left(R_{p}\right)$ were chosen for providing good contact with a fabric surface. The experiments showed that location of too small particles $\left(R_{p}=Z_{p}<0.1\right)$ at the contact moment with fabric was not stable even when settling rate was conservative (up to $1-2 \mathrm{~m} / \mathrm{s}$ ).

The metallic particle falling on the fabric surface had solid structure and did not become deformed because the particle rate did not exceed $1.5 \mathrm{~m} / \mathrm{s}$ when it contacted with fabric surface. Fabric shape did not change too. The experiments were carried out in well-repeatable conditions.

The metallic disk is heated to the high temperature in heating furnace [23] with function of internal volume temperature stabilization (up to $\Theta_{p}=1.473$ ) during a long time (Figure 2).

The moment of contact between hot particle and fabric surface was fixed automatically by flame registration system. It consists of radiation source and detector. A light beam between radiation source and detector was blocked at the particle falling. The first signal via the analog to digital converter comes to the personal computer. The moment of liquid fuel evaporation products ignition was registered by photo cell. It formed a repeated signal at flame appearance. The second signal was fixed by the personal computer too. Time period between two signals characterized ignition delay time $\left(t_{d}\right)$ in the system "fabric_combustible liquidoxidant" (Figure 1).

The error of hot particle temperature measurement estimated by the methods [24] did not exceed 1-2\%. It has been established that the temperature of a particle decreases less than 3-4 K during falling, because the error of temperature determination was less than $\pm 0.5 \%$. This deviation can be neglected in the analysis because the particle temperature was more than $900 \mathrm{~K}$ at experiments. Systematic inaccuracy of time-keeping was $\pm 0.005 \%$ and corresponded to computer possibilities.

Variable error of ignition delay time determination was calculated according to the results of experiment. Eight experiments were carried out for each particle rate at the fixed value of $\Theta_{p}$. The average values of $\tau_{d}$ and mean-square 
deviations $(S)$ were determined by the methods [24] for each value of $\Theta_{p}$. The values of $S$ were differing (from $\pm 0.01 \mathrm{~s}$ to $\pm 0.17 \mathrm{~s}$ ) for each type of studied fabrics and combustible liquids. Corresponding variation coefficients ranged from $\pm 4.5 \%$ to $\pm 15 \%$. Calculated mean-square deviations and variation coefficients are acceptable for conducted experiments due to realization of complication mechanism for studied process.

Visual observations over the ignition of heterogeneous structures were insufficient for adequate description of process mechanism. So the video recording [23] with frequency at 50 frames per second was used for details studied mechanisms and allocation rather subtle effects after repeated analysis of video frames.

\section{Results and Discussion}

The numerical simulation was carried out for the following values of thermophysical and thermochemical parameters:

gasoline: $C=2060 \mathrm{~J} /(\mathrm{kg} \cdot \mathrm{K}), \rho=751 \mathrm{~kg} / \mathrm{m}^{3}, \lambda=$ $0.116 \mathrm{~W} /(\mathrm{m} \cdot \mathrm{K}), Q_{e}=29.4 \cdot 10^{3} \mathrm{~J} / \mathrm{kg}$, and $M=$ $100 \mathrm{~kg} / \mathrm{kmol}$;

gasoline vapors: $C=2280 \mathrm{~J} /(\mathrm{kg} \cdot \mathrm{K}), \rho=2.5 \mathrm{~kg} / \mathrm{m}^{3}$, $\lambda=0.027 \mathrm{~W} /(\mathrm{m} \cdot \mathrm{K}), Q_{o}=45 \cdot 10^{6} \mathrm{~J} / \mathrm{kg}, E=130$. $10^{3} \mathrm{~J} / \mathrm{mole}, k_{0}=7 \cdot 10^{6} \mathrm{~s}^{-1}, \beta=0.0012 \mathrm{~K}^{-1}$, and $D=$ $14.12 \cdot 10^{-6} \mathrm{~m}^{2} / \mathrm{s} ; v=1.21 \cdot 10^{9} \mathrm{~m}^{2} / \mathrm{s}$;

kerosene: $C=2190 \mathrm{~J} /(\mathrm{kg} \cdot \mathrm{K}), \rho=885 \mathrm{~kg} / \mathrm{m}^{3}$, $\lambda=0.117 \mathrm{~W} /(\mathrm{m} \cdot \mathrm{K}), Q_{e}=26.1 \cdot 10^{3} \mathrm{~J} / \mathrm{kg}$, and $M=$ $166.2 \mathrm{~kg} / \mathrm{kmol}$;

kerosene vapors: $C=2370 \mathrm{~J} /(\mathrm{kg} \cdot \mathrm{K}), \rho=2.8 \mathrm{~kg} / \mathrm{m}^{3}$, $\lambda=0.028 \mathrm{~W} /(\mathrm{m} \cdot \mathrm{K}), Q_{o}=43.8 \cdot 10^{6} \mathrm{~J} / \mathrm{kg}, E=190$. $10^{3} \mathrm{~J} / \mathrm{mole}, k_{0}=7 \cdot 10^{7} \mathrm{~s}^{-1}, \beta=0.00096 \mathrm{~K}^{-1}$, and $D=$ $8.07 \cdot 10^{-6} \mathrm{~m}^{2} / \mathrm{s} ; v=0.66 \cdot 10^{9} \mathrm{~m}^{2} / \mathrm{s}$;

diesel fuel: $C=2980 \mathrm{~J} /(\mathrm{kg} \cdot \mathrm{K}), \rho=887.7 \mathrm{~kg} / \mathrm{m}^{3}$, $\lambda=0.1169 \mathrm{~W} /(\mathrm{m} \cdot \mathrm{K}), Q_{e}=25 \cdot 10^{3} \mathrm{~J} / \mathrm{kg}$, and $M=$ $150 \mathrm{~kg} / \mathrm{kmol}$;

diesel fuel vapors: $C=3230 \mathrm{~J} /(\mathrm{kg} \cdot \mathrm{K}), \rho=3.1 \mathrm{~kg} / \mathrm{m}^{3}$, $\lambda=0.029 \mathrm{~W} /(\mathrm{m} \cdot \mathrm{K}), Q_{o}=42 \cdot 10^{6} \mathrm{~J} / \mathrm{kg}, E=250$. $10^{3} \mathrm{~J} / \mathrm{mole}, k_{0}=9 \cdot 10^{8} \mathrm{~s}^{-1}, \beta=0.0009 \mathrm{~K}^{-1}$, and $D=$ $5.29 \cdot 10^{-6} \mathrm{~m}^{2} / \mathrm{s} ; v=1.15 \cdot 10^{9} \mathrm{~m}^{2} / \mathrm{s}$;

wool: $C=1721 \mathrm{~J} /(\mathrm{kg} \cdot \mathrm{K}), \rho=1320 \mathrm{~kg} / \mathrm{m}^{3}$, and $\lambda=$ $0.052 \mathrm{~W} /(\mathrm{m} \cdot \mathrm{K})$;

silk: $C=1386 \mathrm{~J} /(\mathrm{kg} \cdot \mathrm{K}), \rho=1560 \mathrm{~kg} / \mathrm{m}^{3}$, and $\lambda=$ $0.06 \mathrm{~W} /(\mathrm{m} \cdot \mathrm{K})$;

flax: $C=1580 \mathrm{~J} /(\mathrm{kg} \cdot \mathrm{K}), \rho=1500 \mathrm{~kg} / \mathrm{m}^{3}$, and $\lambda=$ $0.088 \mathrm{~W} /(\mathrm{m} \cdot \mathrm{K})$;

steel: $C=470 \mathrm{~J} /(\mathrm{kg} \cdot \mathrm{K}), \rho=7831 \mathrm{~kg} / \mathrm{m}^{3}, \lambda=$ $49 \mathrm{~W} /(\mathrm{m} \cdot \mathrm{K})$, and $Q_{c}=205 \cdot 10^{3} \mathrm{~J} / \mathrm{kg}$;

oxidant (air): $C=1006 \mathrm{~J} /(\mathrm{kg} \cdot \mathrm{K}), \rho=1.161 \mathrm{~kg} / \mathrm{m}^{3}$, and $\lambda=0.026 \mathrm{~W} /(\mathrm{m} \cdot \mathrm{K})$.

Particle rates $R_{p}=0.15-0.25$ and $Z_{p}=0.15-0.25$; fabric thicknesses $Z_{1}=0.15$; domain solution rates $R_{L}=Z_{L}=1$; particle initial temperature $\Theta_{p}=1-1.5$; fabric and oxidant initial temperature $\Theta_{0}=0.3$; absolute gas constant $R_{t}=$ $8.31 \mathrm{~J} /(\mathrm{mol} \cdot \mathrm{K})$.

Executed earlier numerical investigations results of ignition processes in the heterogeneous system "hot particlefabric impregnated by combustible liquid-gas mixture" [15] show that volume fraction of combustible liquid vapors in fabric $\varphi$ and heat content of ignition source (that depends on the temperature $\Theta_{p}$ and rates $R_{p}, Z_{p}$ ) are the basic parameters, which have bigger effect on the necessary and sufficient ignition conditions performance. Therefore it is interesting to estimate influence of these parameters on ignition integrated characteristics.

At numerical simulation the volume fraction value $\varphi$ was varied within the range from 5 up to $50 \%$ in conditions of fabric fixed thickness $Z_{1}$. The value $\varphi$ was controlled by fabric weighing before and after impregnation. Numerically and experimentally was established that the stable ignition of the heterogeneous system (Figure 1) takes place under fabric high-porous structure (up to $30-40 \%$ of the heterogeneous system mass is the liquid condensed substance). So regardless of the local source temperature $\Theta_{p}$ and rates $R_{p}, Z_{p}$, it can be concluded that the requirement $\varphi>30 \%$ should be accomplished for ignition realization in the system (Figure 1).

It is significant that the established limit value $\varphi$ is less than in [15]. It can be explained by the fact that the local energy supply from particle to fabric with thickness $Z_{1}>$ 0.25 leads to warming up only and sufficiently thin nearsurface layer [15]. So small portion of the liquid takes part in the endothermic phase transition. As a result of the executed numerical investigations it was established that thin $\left(Z_{1}<0.15\right)$ fabrics can become warm even on all thicknesses of $Z_{1}$. It depends on temperature and the power source rates. Therefore blowing vapor from the fabric surface to oxidizer environment is more intense than it was supposed in statements [15]. As a consequence, a few smaller values of combustible liquid concentration in fabric are necessary for ignition realization in system (Figure 1) in comparison with results [15].

The dependencies of ignition integrated characteristics (time $\tau_{d}$ ) on temperature and rate $R_{p}$ (with fixed $Z_{p}$ ) of metallic particle for fabrics impregnated by different combustible liquids (gasoline, kerosene, and diesel fuel) are present on Figures 3 and 4 . We used mathematical model (2)-(8) for calculating these characteristics. Earlier it was established [5, 6] that correlation of the square of local power source contact with the condensed substance and square of gas medium determined the possibility of ignition conditions realization. Therefore the particle crosswise size $R_{p}$ that determined its contacting area with fabric was varied.

Figure 3 shows the experimental values $\tau_{d}$ and approximate curve obtained by the least square method. There is good correlation of executed numerical and experimental investigation results. Deviations of experimental values $\tau_{d}$ (on the approximate curve) rather corresponding calculated values do not exceed $18 \%$. With temperature increasing $\Theta_{p}$ these deviations are reduced to $11 \%\left(\right.$ at $\left.\Theta_{p}=1.45\right)$. This feature can be explained by the fact that we used known [25-27] values of oxidation reactions kinetic parameters 


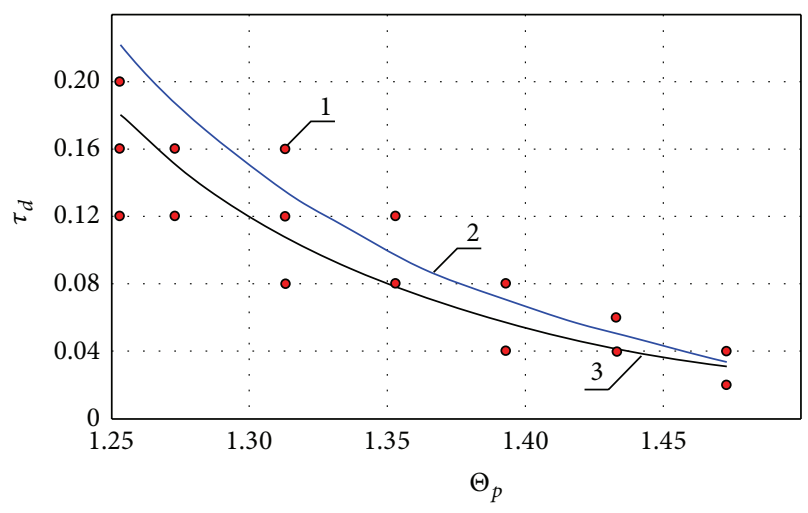

FIGURE 3: Dimensionless ignition delay time versus dimensionless initial temperature of the energy source: 1 -experimental values, 2-theoretical values, and 3-approximating curve for experimental points.

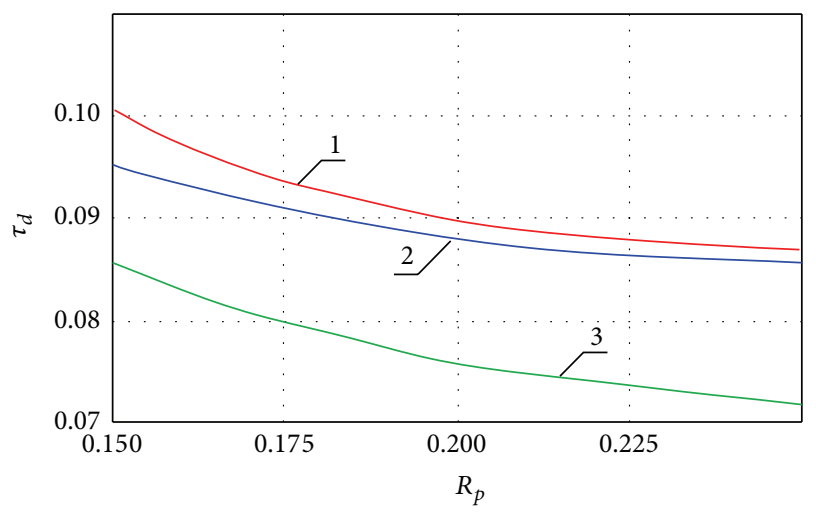

FIGURE 4: Dimensionless ignition delay time versus rate of a heating source $R_{p}$ at $Z_{p}=0.4$ and $\Theta_{p}=1.35: 1$ - diesel fuel, 2-kerosene, and 3-gasoline.

( $k_{0}=$ const and $E=$ const $)$ at the simulation. For approximating the theoretical models to real applications it is advisable to take into account the dependence of $k_{0}$ and $E$ on the temperature according to [28]. It is known [28] that this feature is important at the local heating of small droplets, thin films, and large amounts of liquid condensed substances by sources with limited power consumption. The similar task requires special consideration. Therefore physical and chemical processes features associated with dependencies $k_{0}$ $=f\left(\Theta_{p}\right)$ and $E=f\left(\Theta_{p}\right)$ are not analyzed in this paper.

Comparison of dependences $\tau_{d}=f\left(\Theta_{p}\right)$ shown in Figures 3 and 4 with results of investigations [15] allows drawing a conclusion that the ignition delay time for fabrics with small thickness $Z_{1}$ (Figure 1) is less in several times than analog characteristic for fabric with thicknesses essentially bigger than heating source rates [15]. It can be explained by the intensification of heat fabric process with decreasing $Z_{1}$. As a consequence the injection of evaporation products increases near the heating source. The smaller quantity of energy of a local source is spent for heterogeneous structure warming up and more quantity of energy is used on warming up of oxidizer with fuel vapors mix. It leads to decreasing of heat time and oxidation reaction acceleration.

Dependences (Figure 4) illustrate that the source rates (especially $R_{p}$ ) have less impact on integrated ignition characteristics compared to $\Theta_{p}$ (Figure 3$)$. Therefore even a relatively small area of particle contact with the impregnated fabric is sufficient to achieve the high velocities of the phase transition and the oxidation reaction acceleration (when $\left.\Theta_{p}>1\right)$. At the same time the dependences of evaporation velocity $W_{e}$ and oxidation velocity $W_{o}$ on the temperature are nonlinear. Therefore, even a small $( \pm 0.1)$ change of $\Theta_{p}$ affects $\tau_{d}$ significantly.

For dependencies shown in Figures 3 and 4 the approximation expressions were obtained:

$$
\begin{aligned}
& \tau_{d}=7.271-9.719 \Theta_{p}+3.264 \Theta_{p}^{2} \text { at } 1.25<\Theta_{p}<1.5, R_{p} \\
& =0.15 \text { and } Z_{p}=0.25 ; \\
& \tau_{d}=0.147-0.579 R_{p}+1.116 R_{p}^{2} \text { at } 0.15<R_{p}<0.25, Z_{p} \\
& =0.25 \text { and } \Theta_{p}=1.35 \text {, combustion liquid-gasoline; } \\
& \tau_{d}=0.145-0.481 R_{p}+0.976 R_{p}^{2} \text { at } 0.15<R_{p}<0.25, Z_{p} \\
& =0.25 \text { and } \Theta_{p}=1.35 \text {, combustion liquid-kerosene; } \\
& \tau_{d}=0.178-0.749 R_{p}+1.541 R_{p}^{2} \text { at } 0.15<R_{p}<0.25, Z_{p} \\
& =0.25 \text { and } \Theta_{p}=1.35 \text {, combustion liquid-diesel fuel. }
\end{aligned}
$$

The developed mathematical model allows getting a large group of approximating expressions by varying the basic parameters of the process over a wide range $(0.15<$ $\left.R_{p}<0.25,1<\Theta_{p}<1.5\right)$. However it is impossible to formulate the approximating expressions taking into account the dependences of the ignition delay time $\tau_{d}$ on initial source temperature $\Theta_{p}$ and its rates $R_{p}$ and $Z_{p}$ corresponding to experimental and theoretical results. Due to the fact that the more parameters or factors that are taken into account at the approximation expressions making the greater approximation error it is expedient to get an approximation expression of the forms $\tau_{d}=f\left(\Theta_{p}\right)$ and $\tau_{d}=f\left(R_{p}\right)$.

The difference between ignition integrated characteristics of fabric impregnated by some kinds of combustible liquids (gasoline, kerosene, and diesel fuel) was established as a result of numerical and experimental investigations of ignition processes in system (Figure 1). These characteristics correspond well to the results of experimental investigations of ignition process in homogeneous system (liquid fuels) [23]. It was determined that the ignition delay time for systems with gasoline vapors is minimal and for systems with diesel fuel vapors is maximal (Figure 4) under otherwise equal conditions. Values of $\tau_{d}$ for systems with kerosene vapors have some intermediate values (Figure 4). It is caused by the evaporation regularities (the phase transition temperature, kinetics, chemical composition, etc.) for different fuels [23]. In experiments with the fabrics impregnated by gasoline it was established that the conditions (by concentration $C_{f}$ ) sufficient for ignition (in small fabric vicinity) are carried out even before the particle falling on the surface of heterogeneous structure. The particle and fabric contact leaded to temperature increase in reaction zone. The stable ignition of fabrics impregnated by kerosene and diesel fuel 


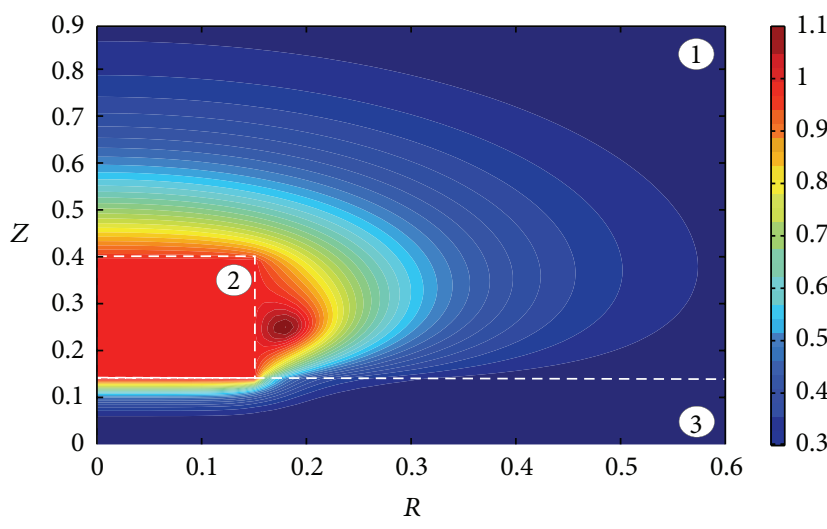

(a)

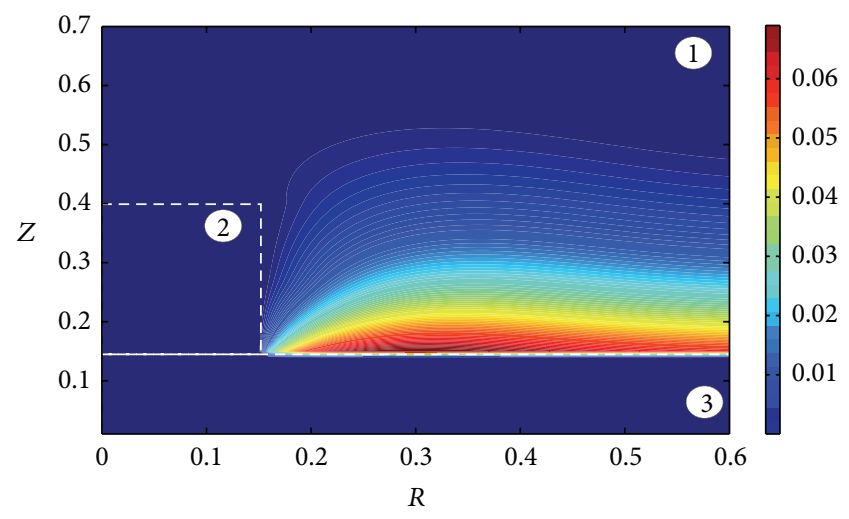

(b)

FIGURE 5: Isotherms $\Theta(a)$ and isolines of fuel concentration $C_{f}(\mathrm{~b})$ at the system "steel particle-the woolen fabric impregnated by keroseneair" at ignition $\left(\tau_{d}=0.314\right)$ for $\Theta_{p}=1.15, R_{p}=0.15, Z_{p}=0.25$, and $\varphi=0.35$ : 1 -gas mixture, 2 - metal particle, and 3-fabric impregnated by liquid combustible substance.

took place only under relative long-lived contact with hot particle (necessary conditions on $C_{f}$ and $\Theta_{p}$ were achieved in great times in comparison with gasoline). At the same time the experimental results showed that ignition happened consistently for all types of combustible liquids in spite of the integrated characteristics difference.

The numerical analysis of ignition modes characterized by the ignition delay time and location of oxidation reaction (the relative contact border of ignition source and substance surface) was accomplished for system (Figure 1) similar as in $[5,6,8]$. It was established that realization of three ignition modes is possible for the system "fabric_combustible liquid-oxidant" (Figure 1) during the local heating. However in contrast to the homogeneous liquid fuels $[5,6,8]$, the mode when zone of exothermal reaction formulates near the lateral sides of ignition source (Figure 5) is the most typical at variation of $\Theta_{p}, R_{p}, Z_{p}$, and $\varphi$ in wide ranges. It can be explained according to the fact that the contact square of the heating source with a substance in the system "fabric-combustible liquid-oxidant" is less than in the system "combustible liquid-oxidant" (particle immersion happens in liquids and square of heat sink into near-surface layer increases). Therefore the local energy source cools down more intensively at interaction with liquid in comparison with the system (Figure 1). The temperatures (Figure 5(a)) and concentrations (Figure 5(b)) sufficient to ignition are reached near the lateral sides of hot particle.

Videograms illustrated that realization of ignition conditions and burning absence are shown in Figures 6 and 7 appropriately. It was established that at the particle temperature $\Theta_{p}<1$ the ignition does not take place in system (Figure 1). The intensive evaporation (Figure 7) without oxidation reaction and subsequent flame appearance are implemented. The experimental results showed that ignition happened consistently at $\Theta_{p}>1$ (Figure 6). Thus $\Theta_{p}=1$ is the limit (low) temperature of steel particle $\left(R_{p}=0.15\right.$ and $\left.Z_{p}=0.25\right)$ at which ignition happens. In case of increasing $R_{p}$ and $Z_{p}$ the limit value of $\Theta_{p}$ decreases but does not reach 0.95 under any circumstances. The limit value of $\Theta_{p}$

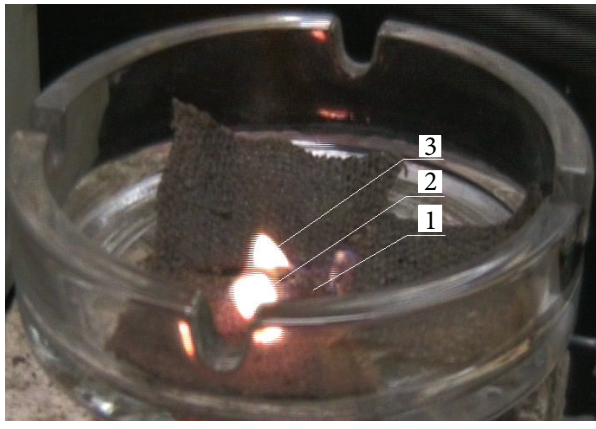

FIGURE 6: Frame of experiment with ignition $\left(\Theta_{p}=1.25, R_{p}=0.15\right.$, and $\left.Z_{p}=0.25\right)$ : 1 -woolen fabric impregnated by kerosene, 2 - steel particle, and 3-flame.

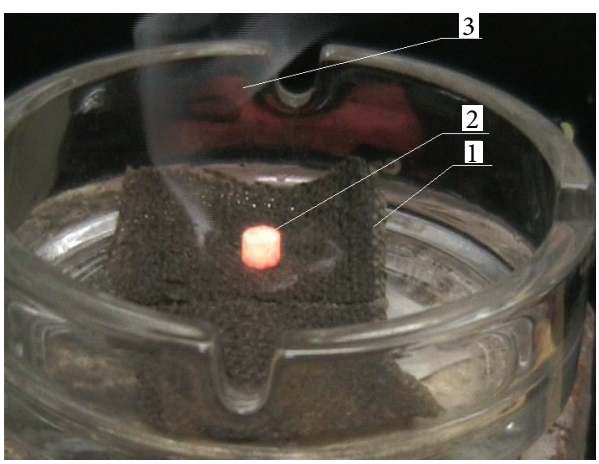

FIGURE 7: Frame of experiment without ignition $\left(\Theta_{p}=1, R_{p}=0.15\right.$, and $\left.Z_{p}=0.25\right)$ : 1-woolen fabric impregnated by kerosene, 2 - steel particle, and 3-smoke without flame.

could be reduced at the fabric thickness $Z_{1}$ decreasing and combustible liquid volume fraction $\varphi$ increasing. However, in this case, it is difficult to provide the conditions for the system output on stationary combustion mode (without the flares and subsequent extinction). 


\section{Conclusions}

The necessary $(\varphi>30 \%)$ and sufficient $\left(\Theta_{p}>1\right)$ ignition conditions of heterogeneous system, widely used fabrics (wool, silk, and flax) impregnated by typical combustible liquids (gasoline, kerosene, and diesel fuel) under the local heating, were established as a result of numerical and experimental investigations. The impact extents of temperature and rates of local energy source and combustible liquid volume fraction in fabric on the ignition integrated characteristics were determined. It was established that the values of $\Theta_{p}$ and $\varphi$ have dominant role in combustion process. Besides it was shown that the main integrated characteristics (ignition delay time) for systems with thin high-porous fabrics can be significantly less than analog characteristic for large thickness fabrics [15].

Besides the good correspondence of numerical and experimental results (numerical simulation assumptions were proved and developed physical and mathematical models were confirmed) was illustrated. Deviations of numerical and experimental values of $\tau_{d}$ did not exceed $18 \%$ under condition of the main processes parameters variation in wide ranges. The good correlation of investigation results with regularities [23] (about modes and ignition mechanisms) for homogeneous structures (liquid fuels) was established.

Investigation results allow making conclusion about the high possibility of ignition conditions realization at cooperation of typical local heat sources with heterogeneous structures, in particular, fabrics impregnated by liquid condensed substances. In the case of a small fabric thickness and its high porosity the ignition process is characterized by small $\left(\tau_{d}<0.1\right)$ ignition delay time close to similar characteristic for homogeneous liquid fuels.

\section{Nomenclatures and Units}
A: Accommodation coefficient
$C$ : Specific heat capacity, $\mathrm{J} /(\mathrm{kg} \cdot \mathrm{K})$
$C_{f}$ : Dimensionless mass fuel vapors
concentration in gas mixture
$\begin{aligned} C_{o}: & \text { Dimensionless mass oxidizer } \\ & \text { concentration in gas mixture } \\ \text { D. } & \text { Coefficient of diffusion } \mathrm{m}^{2} / \mathrm{s}\end{aligned}$
$D:$ Coefficient of diffusion, $\mathrm{m}^{2} / \mathrm{s}$
$E: \quad$ Activation energy, $\mathrm{J} / \mathrm{mol}$
Fo: Fourier number
$g:$ Gravitational acceleration, $\mathrm{m} / \mathrm{s}^{2}$
$k_{0}$ : Preexponential factor, $\mathrm{s}^{-1}$
$M$ : Molecular mass of liquid, $\mathrm{kg} / \mathrm{kmol}$
$P$ : Pressure of vapors above fabric surface, $\mathrm{N} / \mathrm{m}^{2}$
$P^{n}$ : Saturation pressure of vapors, $\mathrm{N} / \mathrm{m}^{2}$
Pr: Prandtl number
$Q_{c}$ : Heat of particle material crystallization, $\mathrm{J} / \mathrm{kg}$
$Q_{e}:$ Heat of liquid evaporation, J/kg
$Q_{o}$ : Heat of oxidation reaction of fuel vapors in air, $\mathrm{J} / \mathrm{kg}$

$r, z: \quad$ Cylindrical coordinates, $\mathrm{m}$

$r_{L}, z_{L}$ : Solution area rates, $\mathrm{m}$

$r_{p}, z_{p}:$ Particle rates, $\mathrm{m}$

$R, Z: \quad$ Dimensionless analogues of $r, z$

$R_{L}, Z_{L}$ : Dimensionless analogue of $r_{L}, z_{L}$

$R_{p}, Z_{p}$ : Dimensionless analogue of $r_{p}, z_{p}$

$R_{t}: \quad$ Absolute gas constant, $\mathrm{J} /(\mathrm{mol} \cdot \mathrm{K})$

Re: Reynolds number

$S$ : Mean-square deviations

Sc: Schmidt number

Sh: Strouhal number

$t$ : Time, $\mathrm{s}$

$t_{d}: \quad$ Ignition delay time, $s$

$t_{m}: \quad$ Time scale, $s$

T: $\quad$ Temperature, $\mathrm{K}$

$\Delta T: \quad$ Temperature differential $\left(\Delta T=T_{m}-T_{0}\right), \mathrm{K}$

$T_{\mathrm{dr}}: \quad$ Fabric surface temperature, $\mathrm{K}$

$T_{m}: \quad$ Temperature scale, $\mathrm{K}$

$T_{0}$ : Initial temperature, $\mathrm{K}$

$u, v: \quad$ Rates of gas mixture in a projection to axes $r$ and $z, \mathrm{~m} / \mathrm{s}$

$U, V: \quad$ Dimensionless analogue of $u, v$

$V_{c}$ : Linear rate of particle material

$V_{m}: \quad$ Crystallization, $\mathrm{m} / \mathrm{s}$

$W_{c}: \quad$ Mass rate of particle material crystallization, $\mathrm{kg} /\left(\mathrm{m}^{2} \cdot \mathrm{s}\right)$

$W_{e}: \quad$ Mass rate of liquid evaporation, $\mathrm{kg} /\left(\mathrm{m}^{2} \cdot \mathrm{s}\right)$

$W_{o}$ : Mass rate of fuel vapors oxidation in air, $\mathrm{kg} /\left(\mathrm{m}^{3} \cdot \mathrm{s}\right)$.

Greek Symbols

$\Theta:$ Dimensionless temperature

$\Theta_{0}$ : Dimensionless initial temperature of air and fabric

$\Theta_{p}$ : Dimensionless initial temperature of particle

$\Psi$ : Dimensionless stream function analogue

$\Omega:$ Dimensionless analogue of vortex velocity vector

$\beta$ : Coefficient of thermal expansion, $\mathrm{K}^{-1}$

$\lambda$ : Thermal conductivity, $\mathrm{W} /(\mathrm{m} \cdot \mathrm{K})$

$\rho:$ Density, $\mathrm{kg} / \mathrm{m}^{3}$

$\tau$ : Dimensionless time

$\tau_{d}$ : Dimensionless ignition delay time

$v$ : Coefficient of kinematic viscosity, $\mathrm{m}^{2} / \mathrm{s}$

$\varphi$ : Volume fraction

$\psi$ : Stream function, $\mathrm{m}^{3} / \mathrm{s}$

$\psi_{0}$ : Scale of stream function, $\mathrm{m}^{3} / \mathrm{s}$

$\omega$ : Vortex velocity vector, $1 / \mathrm{s}$

$\omega_{0}$ : Scale of vortex velocity vector, $1 / \mathrm{s}$.

\section{Subscripts}

1: Gas mixture

2: Metallic particle

3: Fabric impregnated by combustible liquid

4: Fuel vapor. 


\section{Conflict of Interests}

The authors declare that there is no conflict of interests regarding the publication of this paper.

\section{Acknowledgments}

The reported study was partially supported by Grant of the President of the Russian Federation (Project no. MK2391.2014.8) and State task "Nauka" (code of the federal target scientific and technical Program no. 2.1321.2014).

\section{References}

[1] I. G. Khaneft and A. V. Khaneft, "Effect of the duration of the front edge of the voltage pulse on the electric breakdown of ammonium perchlorate," Technical Physics, vol. 45, no. 4, pp. 423-426, 2000.

[2] V. A. Babuk, "Properties of the surface layer and combustion behavior of metallized solid propellants," Combustion, Explosion and Shock Waves, vol. 45, no. 4, pp. 486-494, 2009.

[3] R. S. Burkina and E. A. Mikova, "High-temperature ignition of a reactive material by a hot inert particle with a finite heat reserve," Combustion, Explosion and Shock Waves, vol. 45, no. 2, pp. 144-150, 2009.

[4] D. O. Glushkov and P. A. Strizhak, "Heat and mass transfer at ignition of solid condensed substance with relatively low calorific power by a local energy source," Journal of Engineering Thermophysics, vol. 21, no. 1, pp. 69-77, 2012.

[5] G. V. Kuznetsov and P. A. Strizhak, "Numerical solution of the problem of ignition of a combustible liquid by a single hot particle," Combustion, Explosion and Shock Waves, vol. 45, no. 5, pp. 42-50, 2009.

[6] G. V. Kuznetsov and P. A. Strizhak, "Effect of the shape of a particle heated to a high temperature on the gas-phase ignition of a liquid film," Russian Journal of Physical Chemistry B, vol. 4, no. 2, pp. 249-255, 2010.

[7] R. Finesso and E. Spessa, "Ignition delay prediction of multiple injections in diesel engines," Fuel, vol. 119, pp. 170-190, 2014.

[8] D. O. Glushkov and P. A. Strizhak, "Transient heat and mass transfer of liquid droplet ignition at the spreading over the heated substrate," Advances in Mechanical Engineering, vol. 2014, Article ID 269321, 9 pages, 2014.

[9] R. M. Aseeva and V. A. Grygorovskaya, "Thermal properties and flammability of polyarylenes and their modifying products," Polymer Degradation and Stability, vol. 64, no. 3, pp. 457-463, 1999.

[10] N. S. Zubkova, M. A. Tyuganova, I. S. Reshetnikov, and N. A. Khalturinskii, "Decreasing the combustibility of polyolefins using microencapsulated fire retardants," Fibre Chemistry, vol. 29, no. 3, pp. 166-168, 1997.

[11] L. Shi and M. Y. L. Chew, "Fire behaviors of polymers under autoignition conditions in a cone calorimeter," Fire Safety Journal, vol. 61, pp. 243-253, 2013.

[12] K. Okamoto, M. Hiramatsu, H. Miyamoto et al., "Evaporation and diffusion behavior of fuel mixtures of gasoline and kerosene," Fire Safety Journal, vol. 49, pp. 47-61, 2012.

[13] D. K. Srivastava, E. Wintner, and A. K. Agarwal, "Effect of focal size on the laser ignition of compressed natural gas-air mixture," Optics and Lasers in Engineering, vol. 58, pp. 67-79, 2014.
[14] D. K. Srivastava and A. K. Agarwal, "Comparative experimental evaluation of performance, combustion and emissions of laser ignition with conventional spark plug in a compressed natural gas fuelled single cylinder engine," Fuel, vol. 123, pp. 113-122, 2014.

[15] G. V. Kuznetsov and P. A. Strizhak, "Heat and mass transfer in hot-particle-induced ignition of a liquid-fuel vapor entering the ambient air from the surface of fabric impregnated with the fuel," Journal of Engineering Physics and Thermophysics, vol. 82, no. 3, pp. 448-455, 2009.

[16] G. V. Kuznetsov and P. A. Strizhak, "3D problem of heat and mass transfer at the ignition of a combustible liquid by a heated metal particle," Journal of Engineering Thermophysics, vol. 18, no. 1, pp. 72-79, 2009.

[17] D. A. Frank-Kamenetsky, Diffusion and Heat Transfer in Chemical Kinetics, Plenum, New York, NY, USA, 1969.

[18] V. N. Vilyunov and V. E. Zarko, Ignition of Solids, Elsevier, Elsevier, Amsterdam, The Netherland, 1989.

[19] H. D. Baehr and K. Stephan, Heat and Mass Transfer, Springer, Berlin, Germany, 1998.

[20] P. J. Roache, Computational Fluid Dynamics, Hermosa Publishers, Albuquerque, NM, USA, 1976.

[21] A. Belmiloudi, Heat Transfer-Mathematical Modelling , Numerical Methods and Information Technology, InTech, Rijeka, Croatia, 2011.

[22] D. Shang, Theory of Heat Transfer with Forced Convection Film Flows, Springer, New York, NY, USA, 2011.

[23] G. V. Kuznetsov, A. V. Zaharevich, and V. I. Maksimov, "About the mechanism of gasoline ignition by single metallic particle heated up to high temperatures," Fire and Explosion Safety, vol. 17, no. 5, pp. 39-42, 2008.

[24] J. P. Buonaccorsi, Measurement Error: Models, Methods, and Applications, Taylor and Francis Group/CRC Press, New York, NY, USA, 2010.

[25] J. House, Principles of Chemical Kinetics, Academic Press, New York, NY, USA, 2007.

[26] V. Patel, Ed., Chemical Kinetics, InTech, Rijeka, Croatia, 2012.

[27] P. Deb, Kinetics of Heterogeneous Solid State Processes, Springer, New York, NY, USA, 2014.

[28] P. Dagaut and M. Cathonnet, "The ignition, oxidation, and combustion of kerosene: a review of experimental and kinetic modeling," Progress in Energy and Combustion Science, vol. 32, no. 1, pp. 48-92, 2006. 


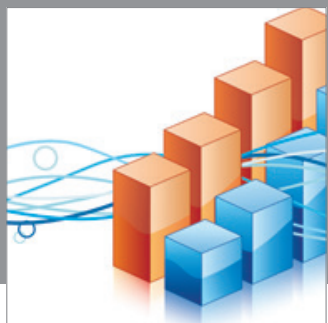

Advances in

Operations Research

mansans

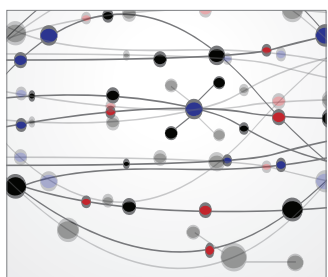

The Scientific World Journal
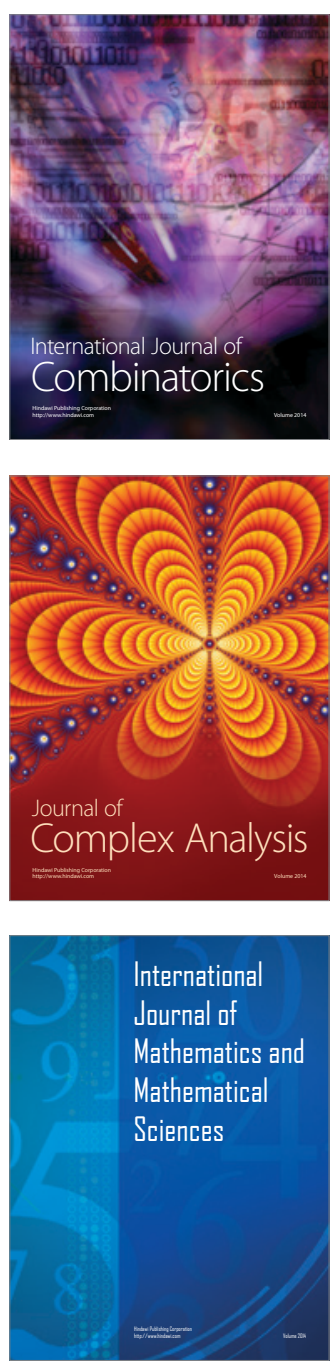
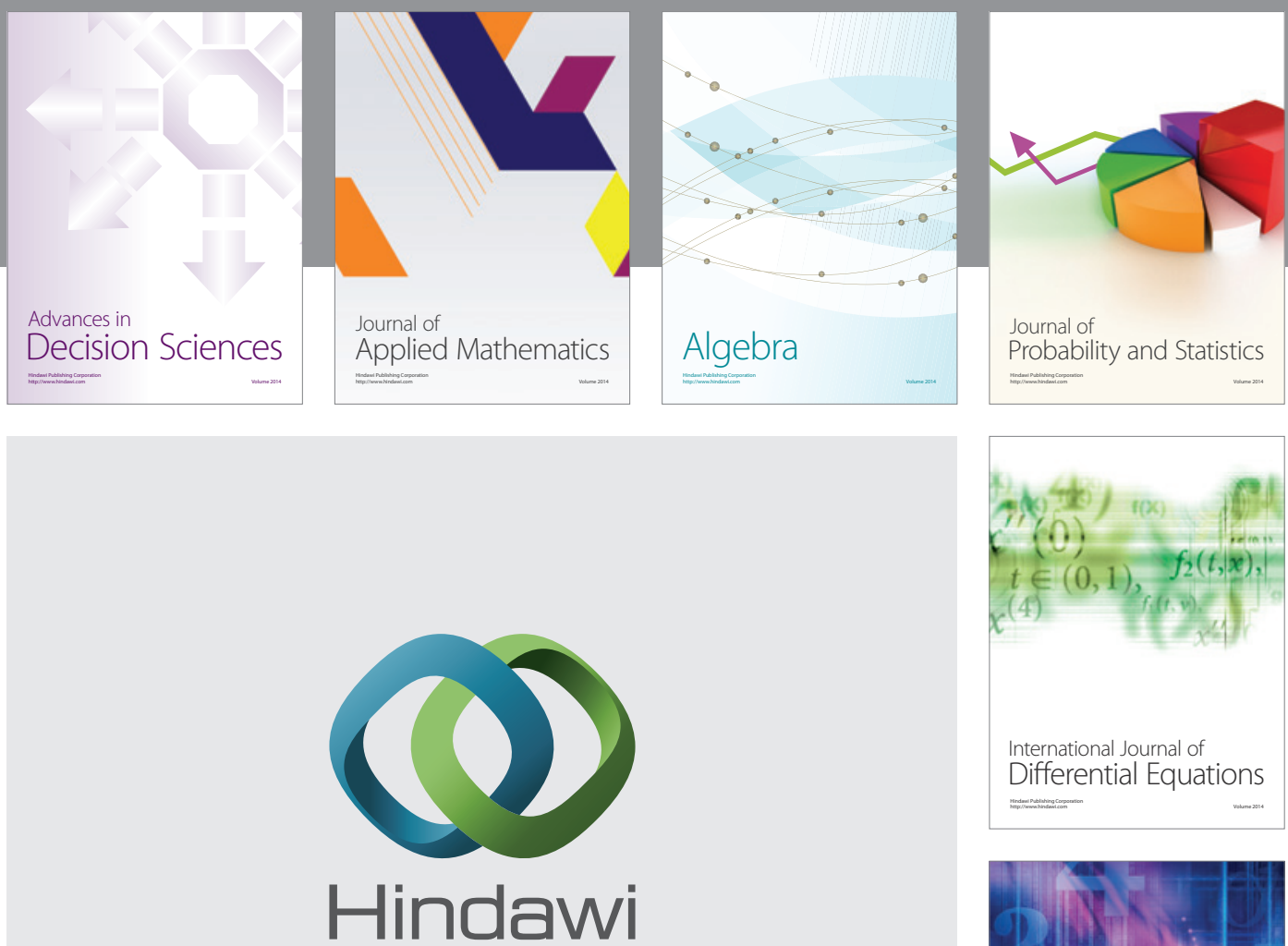

Submit your manuscripts at http://www.hindawi.com
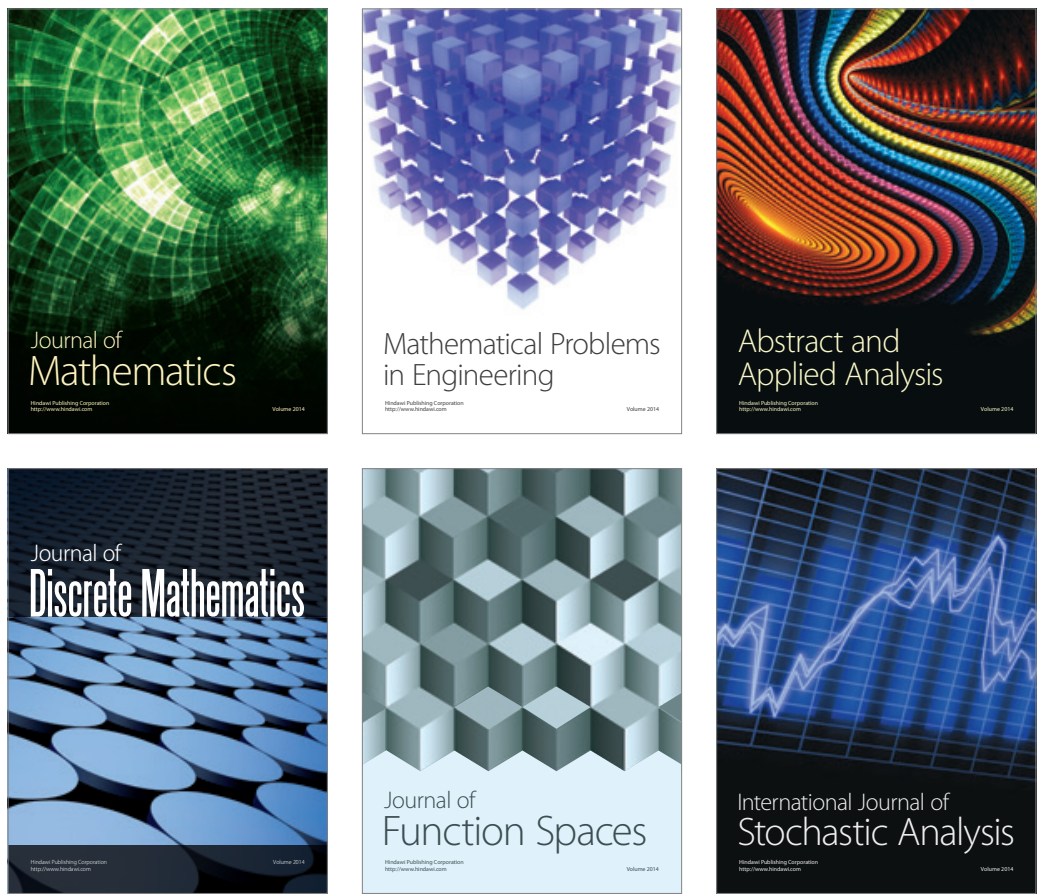

Journal of

Function Spaces

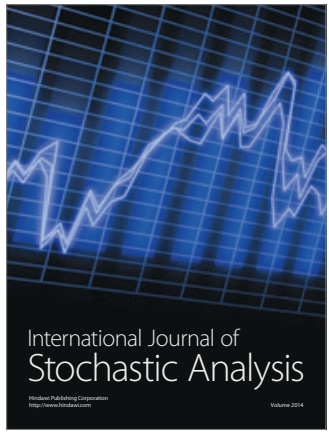

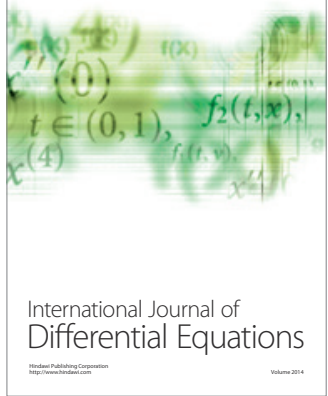
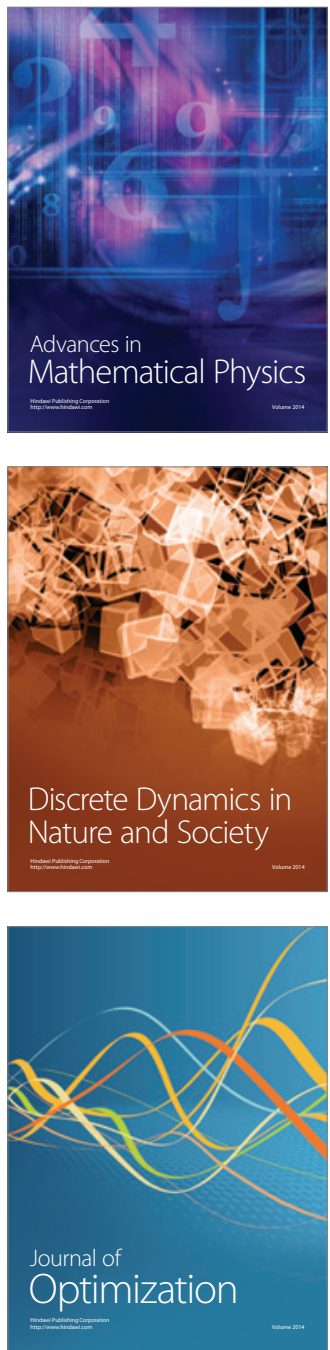